\title{
On the number of zero increments of random walks with a barrier
}

\author{
Alex Iksanov $^{1}$ and Pavlo Negadajlov $\|^{1}$ \\ ${ }^{1}$ Faculty of Cybernetics, National T. Shevchenko University of Kiev, Kiev-01033, Ukraine
}

This is the second submission of this document.

Continuing the line of research initiated in Iksanov and Möhle (2008) and Negadajlov (2008) we investigate the asymptotic (as $n \rightarrow \infty$ ) behaviour of $V_{n}$ the number of zero increments before the absorption in a random walk with the barrier $n$. In particular, when the step of the unrestricted random walk has a finite mean, we prove that the number of zero increments converges in distribution. We also establish a weak law of large numbers for $V_{n}$ under a regular variation assumption.

Keywords: absorption time; recursion with random indices; random walk; undershoot.

\section{Introduction and results}

Let $\left\{\xi_{k}: k \in \mathbb{N}\right\}$ be independent copies of a random variable $\xi$ with proper and non-degenerate distribution

$$
p_{k}:=\mathbb{P}\{\xi=k\}, \quad k \in \mathbb{N}, p_{1}>0 .
$$

Fix $n \in \mathbb{N}$. The sequence $\left\{R_{k}^{(n)}: k \in \mathbb{N}_{0}:=\{0,1, \ldots\}\right\}$ defined as follows: $R_{0}^{(n)}:=0$ and

$$
R_{k}^{(n)}:=R_{k-1}^{(n)}+\xi_{k} 1_{\left\{R_{k-1}^{(n)}+\xi_{k}<n\right\}}, \quad k \in \mathbb{N}
$$

is called a random walk with the barrier $n$. The Markov chain $\left\{R_{k}^{(n)}: k \in \mathbb{N}_{0}\right\}$ is non-decreasing and has the unique absorbing state $n-1$. Denote by

$$
T_{n}:=\inf \left\{k \in \mathbb{N}: R_{k}^{(n)}=n-1\right\}=\sum_{l=1}^{\infty} 1_{\left\{R_{l}^{(n)}<n-1\right\}}+1
$$

the absorption time. Other interesting functionals acting on random walks with a barrier are the number of non-zero increments (jumps)

$$
M_{n}:=\#\left\{k \in \mathbb{N}: R_{k-1}^{(n)} \neq R_{k}^{(n)}\right\}=\sum_{l=0}^{\infty} 1_{\left\{R_{l}^{(n)}+\xi_{l+1}<n\right\}}
$$

\footnotetext{
${ }^{\dagger}$ The research of both authors was supported by the German Scientific Foundation (project no. 436UKR 113/93/0-1). 1365-8050 @ 2008 Discrete Mathematics and Theoretical Computer Science (DMTCS), Nancy, France
} 
and the number of zero increments before the absorption time

$$
V_{n}:=\#\left\{k \in\left[0, T_{n}-1\right]: R_{k-1}^{(n)}=R_{k}^{(n)}\right\}=\sum_{l=0}^{T_{n}-1} 1_{\left\{R_{l}^{(n)}+\xi_{l+1} \geq n\right\}} .
$$

Notice that the last summand of the latter sum always equals zero. We however do not replace the upper limit of the summation by $T_{n}-2$ to avoid summation over the empty set.

Two real-world examples where the random walk with a barrier naturally arises are given next.

(a) An enterprise can pay 500000 Euro per month as a salary to all called-in employees. Potential employees apply for salaries which are independent copies of a random variable $\xi$ taking values 100,200 , $300, \ldots$ Euro. Then $T_{500001}, M_{500001}$ and $V_{500001}$ define the number of people which apply for a job, to be hired and to be turned down, respectively.

(b) A private company (an express, say) specializes in transportation of goods and uses three lorries which altogether are able to transport 10 tones. Assume that requests for transportation are independent copies of a random variable $\xi$ taking values in the set of multiples of $100 \mathrm{~kg}$. Then $T_{10001}, M_{10001}$ and $V_{10001}$ define the total number of requests, the number of requests to be satisfied and to be rejected, respectively.

In [5] and [7] the weak convergence of properly normalized and centered $M_{n}$ and $T_{n}$, respectively, was investigated. Assuming that the distribution of $\xi$ belongs to the domain of attraction of a stable law, a complete characterization of normalizing constants and possible limiting laws was obtained. The class of limiting laws is comprised of stable laws and laws of the integrals $\int_{0}^{\infty} e^{-X_{t}} d t$, where $\left\{X_{t}: t \geq 0\right\}$ is a zero-drift subordinator with known Lévy measure.

Furthermore, in [7] it was proved that all the results obtained in [5] for $M_{n}$ remain valid with $M_{n}$ replaced by $T_{n}$. From this last observation it follows that the number of zero increments $V_{n}=T_{n}-M_{n}$ is "essentially smaller" than the number of non-zero increments $M_{n}$.

The aim of the paper is to investigate asymptotic behaviour of $V_{n}$ as $n \rightarrow \infty$. In Theorem 1.1 it is proved that under the assumption $m:=\mathbb{E} \xi<\infty$ the sequence $\left\{V_{n}: n \in \mathbb{N}\right\}$ converges in distribution. In Theorem 1.2 assuming that $m=\infty$ and that the tail of distribution of $\xi$ is regularly varying at $\infty$, a weak law of large numbers is established. All the proofs rely upon the following observation: marginal distributions of the sequence $\left\{V_{n}: n \in \mathbb{N}\right\}$ satisfy the recursions $[6$ which we call recursions with random indices. It is well-known that recursive structure is typical for many objects arising in the Analysis of Algorithms, in the theory of random graphs, in the coalescent theory, etc. In this paper, we exhibit a relatively new model where recursions appear in a natural way and exploiting them turns out to be $a$ right way of thinking.

Before formulating the main results, notice that Hinderer and Walk [4] investigated processes more general than random walks with a barrier but the circle of problems they considered was different from ours. As far as we know, the term "random walk with a barrier" was introduced in [5].

Theorem 1.1 If $\mathbb{E} \xi<\infty$, then, as $n \rightarrow \infty$,

$$
V_{n} \rightarrow_{d} V:=V_{Y}+1-2 \cdot 1_{\{Y=1\}},
$$

where $Y$ is a random variable with distribution

$$
\mathbb{P}\{Y=k\}=(\mathbb{E} \xi)^{-1} \mathbb{P}\{\xi \geq k\}, k \in \mathbb{N},
$$

which is independent of $\left\{V_{n}: n \in \mathbb{N}\right\}$. In particular, $\mathbb{P}\{V=0\}=(\mathbb{E} \xi)^{-1}$. 
Throughout the paper by $\psi(x):=\Gamma^{\prime}(x) / \Gamma(x)$ we denote the logarithmic derivative of the gamma function. Also, set

$$
m(x):=\int_{0}^{x} \mathbb{P}\{\xi>y\} d y, x>0 .
$$

Theorem 1.2 Suppose that for some $\alpha \in(0,1]$ and some $L$ slowly varying at $+\infty$

$$
\sum_{k=n}^{\infty} p_{k} \sim n^{-\alpha} L(n), \quad n \rightarrow \infty
$$

Then, as $n \rightarrow \infty$,

$$
\frac{V_{n}}{\mathbb{E} V_{n}} \rightarrow_{P} 1
$$

and $\mathbb{E} V_{n} \sim(\psi(1)-\psi(1-\alpha))^{-1} \log n$, if $\alpha \in(0,1)$, and $\mathbb{E} V_{n} \sim \log m(n)$, if $\alpha=1$.

The rest of the paper is organized as follows: in Section 2 we discuss certain recursions with random indices which naturally arise in the context of this work and in Section 3 the proofs of our main results are given.

Notation: the record $r(\cdot) \sim s(\cdot)$ means that $r(\cdot) / s(\cdot) \rightarrow 1$, as an argument goes to $+\infty$; the record $X_{n} \rightarrow_{d}\left(\Rightarrow, \rightarrow_{P}\right) X$ means that the limit relation holds as $n \rightarrow \infty$.

\section{Recursions with random indices}

For fixed $m, i \in \mathbb{N}$ define $\widehat{R}_{0}^{(m)}(i):=0$ and

$$
\widehat{R}_{k}^{(m)}(i):=\widehat{R}_{k-1}^{(m)}(i)+\xi_{i+k} 1_{\left\{\widehat{R}_{k-1}^{(m)}(i)+\xi_{i+k}<m\right\}}, k \in \mathbb{N},
$$

and

$$
\widehat{T}_{m}(i):=\sum_{l=1}^{\infty} 1_{\left\{\widehat{R}_{l}^{(m)}(i)<m-1\right\}}, \widehat{V}_{m}(i):=\sum_{l=0}^{\widehat{T}_{m}(i)-1} 1_{\left\{\widehat{R}_{l}^{(m)}(i)+\xi_{i+l+1} \geq m\right\}} .
$$

For fixed $n \in \mathbb{N}$ and any $i \in \mathbb{N}$ marginal distributions of the sequences $\left\{R_{k}^{(n)}: k \in \mathbb{N}_{0}\right\}$ and $\left\{R_{k}^{(n)}(i)\right.$ : $\left.k \in \mathbb{N}_{0}\right\}$ are the same. Therefore,

$$
\widehat{T}_{n}(i)={ }_{d} T_{n}, \text { AND } \widehat{V}_{n}(i)={ }_{d} V_{n} .
$$

Introduce the notation $S_{0}:=0, S_{n}:=\xi_{1}+\cdots+\xi_{n}$ and

$$
N_{n}:=\inf \left\{k \geq 1: S_{k} \geq n\right\} \text { AND } Y_{n}:=n-S_{N_{n}-1}, n \in \mathbb{N} .
$$

The sequence $\left\{S_{n}: n \in \mathbb{N}_{0}\right\}$ is an unrestricted random walk on the basis of which the random walk with barrier is constructed. The random variable $Y_{n}$ is called undershoot at $n$.

With probability one, we have

$$
T_{n}=\left(1+\widehat{T}_{Y_{n}}\left(N_{n}\right)\right) 1_{\left\{Y_{n} \neq 1\right\}}+N_{n}-1=\widehat{T}_{Y_{n}}\left(N_{n}\right)+N_{n}-2 \cdot 1_{\left\{Y_{n}=1\right\}},
$$


which can be checked as follows. If $n-R_{N_{n}-1}^{(n)}=n-S_{N_{n}-1}=Y_{n}=1$, then $T_{n}=N_{n}-1$, and if $Y_{n} \neq 1$, then $R_{N_{n}}^{(n)}=R_{N_{n}-1}^{(n)}=S_{N_{n}-1}<n-1$. Therefore,

$$
T_{n}=N_{n}-1+\left(1+\sum_{l=1}^{\infty} 1_{\left\{R_{N_{n}+l}^{(n)}<n-1\right\}}\right) 1_{\left\{Y_{n} \neq 1\right\}}=N_{n}-1+\left(1+\widehat{T}_{Y_{n}}\left(N_{n}\right)\right) 1_{\left\{Y_{n} \neq 1\right\}} .
$$

Similarly, with probability one we have

$$
V_{n}=\left(\widehat{V}_{Y_{n}}\left(N_{n}\right)+1\right) 1_{\left\{Y_{n} \neq 1\right\}}=\widehat{V}_{Y_{n}}\left(N_{n}\right)+1-2 \cdot 1_{\left\{Y_{n}=1\right\}}
$$

which can be verified as follows. If $n-R_{N_{n}-1}^{(n)}=n-S_{N_{n}-1}=Y_{n}=1$, then $V_{n}=0$, and if $Y_{n} \neq 1$, then the first non-zero increment of the random walk with barrier is $R_{N_{n}}^{(n)}-R_{N_{n}-1}^{(n)}$, and $T_{n} \geq N_{n}+1$. Therefore,

$$
\begin{gathered}
V_{n}=\left(1+\sum_{l=N_{n}}^{T_{n}-1} 1_{\left\{R_{l}^{(n)}+\xi_{l+1} \geq n\right\}}\right) 1_{\left\{Y_{n} \neq 1\right\}}=\left(1+\sum_{l=0}^{T_{n}-N_{n}-1} 1_{\left\{R_{N_{n}+l}^{(n)}+\xi_{N_{n}+l+1} \geq n\right\}}\right) 1_{\left\{Y_{n} \neq 1\right\}}= \\
\text { BY (4) }=\left(1+\sum_{l=0}^{\widehat{T}_{Y_{n}}\left(N_{n}\right)-1} 1_{\left\{\widehat{R}_{l}^{\left(Y_{n}\right)}\left(N_{n}\right)+\xi_{N_{n}+l+1} \geq Y_{n}\right\}}\right) 1_{\left\{Y_{n} \neq 1\right\}}=\left(1+\widehat{V}_{Y_{n}}\left(N_{n}\right)\right) 1_{\left\{Y_{n} \neq 1\right\}} .
\end{gathered}
$$

¿From (3) and (5) we obtain a recursion with random indices

$$
V_{1}:=1, \quad V_{n}={ }_{d} V_{Y_{n}}^{\prime}+1-2 \cdot 1_{\left\{Y_{n}=1\right\}},
$$

where $Y_{n}$ is independent of the sequence $\left\{V_{k}^{\prime}: k \in \mathbb{N}\right\}$ which is a copy of $\left\{V_{k}: k \in \mathbb{N}\right\}$.

\section{Proofs}

Letting $n \rightarrow \infty$ in the equality of distributions 6 and taking into account the convergence $Y_{n} \rightarrow_{d} Y$ (see, for example, p. 371 in [3]) the result of Theorem 1.1 follows.

To prove Theorem 1.2 we need an auxiliary result.

Lemma 3.1 If (1) holds with $\alpha \in[0,1)$, then, as $n \rightarrow \infty$,

$$
\begin{gathered}
\mathbb{E} \log Y_{n}=\log n-(\psi(1)-\psi(1-\alpha))+o(1) \text { AND } \\
\mathbb{E} \log ^{2} Y_{n}=\log ^{2} n-2(\psi(1)-\psi(1-\alpha)) \log n+o(\log n) .
\end{gathered}
$$

If (17) holds with $\alpha=1$, then, as $n \rightarrow \infty$,

$$
\begin{gathered}
\mathbb{E} \log m\left(Y_{n}\right)=\log m(n)-1+o(1) \text { AND } \\
\mathbb{E} \log ^{2} m\left(Y_{n}\right)=\log ^{2} m(n)-2 \log m(n)+o(\log m(n)) .
\end{gathered}
$$


Proof: If $(1]$ holds with $\alpha \in[0,1)$, then according, for example, to Theorem 8.6 .5 in [1]

$$
\log n-\log Y_{n} \rightarrow_{d}\left(-\log \eta_{\alpha}\right),
$$

where a random variable $\eta_{\alpha}, \alpha \in(0,1)$ has the beta distribution with parameters $1-\alpha$ and $\alpha$, i.e. $\mathbb{P}\left\{\eta_{\alpha} \in d x\right\}=\frac{\sin \pi \alpha}{\pi} x^{-\alpha}(1-x)^{\alpha-1} d x, x \in(0,1)$, and $\mathbb{P}\left\{\eta_{0}=1\right\}=1$.

If we can show that for each $\delta \in(0,1-\alpha)$

$$
\sup _{n \geq 1} \mathbb{E}\left(n / Y_{n}\right)^{\delta}=\sup _{n \geq 1} \mathbb{E} f_{k}\left(\log ^{k}\left(n / Y_{n}\right)\right)<\infty, \quad k \in \mathbb{N},
$$

where $f_{k}(x):=\exp \left(\delta x^{1 / k}\right)$, then by a Vallée-Poussin theorem (see [6]) for each $k \in \mathbb{N}$ the sequence $\left\{\left(\log n-\log Y_{n}\right)^{k}: n \in \mathbb{N}\right\}$ is uniformly integrable. If it is true then $\lim _{n \rightarrow \infty}\left(\log n-\mathbb{E} \log Y_{n}\right)=$ $\mathbb{E}\left(-\log \eta_{\alpha}\right)=\psi(1)-\psi(1-\alpha)<\infty$ which proves 77 , and $\lim _{n \rightarrow \infty} \mathbb{E}\left(\log n-\log Y_{n}\right)^{2}=\mathbb{E} \log ^{2} \eta_{\alpha}=$ $(\psi(1-\alpha)-\psi(1))^{2}+\psi^{\prime}(1-\alpha)-\psi^{\prime}(1)<\infty$, which together with 77 proves 87 .

Let us establish 99. For $j \in \mathbb{N}_{0}$ set $u_{j}:=\sum_{k=0}^{j} \mathbb{P}\left\{S_{k}=j\right\}$. Then

$$
\mathbb{E} Y_{n}^{-\delta}=\sum_{k=0}^{n-1}(n-k)^{-\delta} \mathbb{P}\{\xi \geq n-k\} u_{k} .
$$

The condition 11 implies that $\sum_{k=0}^{n} u_{k} \sim \frac{1}{\Gamma(1-\alpha) \Gamma(1+\alpha)} \frac{n^{\alpha}}{L(n)}$ (see, for example, Theorem 8.7.3 in [1]). The following four relations hold by Corollary 1.7.3 in [1]:

$$
\begin{gathered}
U(s):=\sum_{n=0}^{\infty} s^{n} u_{n} \sim \frac{1}{\Gamma(1-\alpha)} \frac{1}{L\left((1-s)^{-1}\right)(1-s)^{\alpha}} \text { AS } s \uparrow 1 ; \\
V(s):=\sum_{n=1}^{\infty} s^{n} n^{-\delta} \mathbb{P}\{\xi \geq n\} \sim \frac{\Gamma(1-\alpha-\delta) L\left((1-s)^{-1}\right)}{(1-s)^{1-\alpha-\delta}} \text { AS } s \uparrow 1 ; \\
\sum_{n=1}^{\infty} s^{n} \mathbb{E} Y_{n}^{-\delta}=U(s) V(s) \sim \frac{\Gamma(1-\alpha-\delta)}{\Gamma(1-\alpha)} \frac{1}{(1-s)^{1-\delta}} \quad \text { AS } s \uparrow 1 ; \\
\sum_{k=1}^{n} \mathbb{E} Y_{k}^{-\delta} \sim \frac{\Gamma(1-\alpha-\delta)}{\Gamma(1-\alpha) \Gamma(2-\alpha)} n^{1-\delta} .
\end{gathered}
$$

The last equivalence entails 9 .

Assume now that (1]) holds with $\alpha=1$. By Theorem 6 in [2] and its proof,

$$
\log m(n)-\log m\left(Y_{n}\right) \rightarrow_{d}(-\log R),
$$

where $R$ is a random variable with the uniform distribution on $[0,1]$. Therefore, the stated result will be proved if, for example, we can show that the sequences $\left\{\left(\log m(n)-\log m\left(Y_{n}\right)\right)^{k}: n \in \mathbb{N}\right\}, k=1,2$, are uniformly integrable. This latter fact will follows from the following relation: for each $\epsilon \in(0,1)$

$$
\sup _{n \geq 1} \mathbb{E}\left(\frac{m(n)}{m\left(Y_{n}\right)}\right)^{\epsilon}<\infty .
$$


The proof that follows is similar to the previous one. Therefore, we only give a sketch. As before, all the unexplained equivalences connected with asymptotic behaviour of the sequences and corresponding generating functions can be justified by an appeal to Corollary 1.7.3 in [1]. Since $\sum_{k=0}^{n} u_{k} \sim n / m(n)$ (see p. 266 in [2]), then

$$
K(s):=\sum_{n=0}^{\infty} s^{n} u_{n} \sim \frac{1}{\left(m\left((1-s)^{-1}\right)\right)(1-s)} \text { AS } s \uparrow 1 .
$$

Fix any $\epsilon \in(0,1)$. The function $r_{\epsilon}(x):=\int_{0}^{x} m^{-\epsilon}(y) \mathbb{P}\{\xi \geq y\} d y$ is slowly varying at $\infty$, and $\sum_{k=1}^{n} m^{-\epsilon}(k) \mathbb{P}\{\xi \geq k\} \sim r_{\epsilon}(n)$. Consequently,

$$
Z(s):=\sum_{n=1}^{\infty} s^{n} m^{-\epsilon}(n) \mathbb{P}\{\xi \geq n\} \sim r_{\epsilon}\left((1-s)^{-1}\right) \text { AS } s \uparrow 1 .
$$

Hence, as $s \uparrow 1$,

$$
\sum_{n=1}^{\infty} s^{n} \mathbb{E} m^{-\epsilon}\left(Y_{n}\right)=K(s) Z(s) \sim \frac{r_{\epsilon}\left((1-s)^{-1}\right)}{\left(m\left((1-s)^{-1}\right)\right)(1-s)}
$$

and

$$
\sum_{k=1}^{n} \mathbb{E} m^{-\epsilon}\left(Y_{k}\right) \sim \frac{n r_{\epsilon}(n)}{m(n)} .
$$

By L'Hospital's rule, $r_{\epsilon}(n) / m(n) \sim(1-\epsilon)^{-1} m^{-\epsilon}(n)$. Therefore, 11] implies 10].

Proof of Theorem 1.2. Suppose (1) holds with $\alpha \in(0,1)$. Set $a_{n}:=\mathbb{E} V_{n}$ and $b_{n}:=\mathbb{E} V_{n}^{2}$. From (6) we obtain $a_{1}=1$,

$$
a_{n}=\frac{1}{1-\mathbb{P}\left\{Y_{n}=n\right\}}\left(\sum_{k=1}^{n-1} a_{k} \mathbb{P}\left\{Y_{n}=k\right\}+1-2 \mathbb{P}\left\{Y_{n}=1\right\}\right), n=2,3, \ldots
$$

and $b_{1}=1$,

$$
b_{n}=\frac{1}{1-\mathbb{P}\left\{Y_{n}=n\right\}}\left(\sum_{k=1}^{n-1} b_{k} \mathbb{P}\left\{Y_{n}=k\right\}+2 a_{n}-1\right) .
$$

Let us check that

$$
b_{n} \sim a_{n}^{2} \sim k_{\alpha}^{2} \log ^{2} n,
$$

where $k_{\alpha}:=(\psi(1)-\psi(1-\alpha))^{-1}$. Suppose the condition $\varlimsup_{n \rightarrow \infty} \frac{a_{n}}{\log n} \leq k_{\alpha}$ does not hold. Then there exists an $\epsilon>0$ such that the inequalities $a_{n}>\left(k_{\alpha}+\epsilon\right) \log n$ hold for infinitely many values of $n$. It is possible to decrease $\epsilon$ so that the inequality $a_{n}>\left(k_{\alpha}+\epsilon\right) \log n+c$ holds infinitely often for any fixed positive $c$. Thus we can define $n_{c}:=\inf \left\{n \geq 1: a_{n}>\left(k_{\alpha}+\epsilon\right) \log n+c\right\}$. Then

$$
a_{n} \leq\left(k_{\alpha}+\epsilon\right) \log n+c \text { for all } n \in\left\{1,2, \ldots, n_{c}-1\right\} .
$$

¿From (12) it follows that

$$
\left(k_{\alpha}+\epsilon\right) \log n_{c}+c<
$$




$$
\begin{gathered}
<\frac{1}{1-\mathbb{P}\left\{Y_{n_{c}}=n_{c}\right\}}\left(\sum_{i=1}^{n_{c}-1}\left(\left(k_{\alpha}+\epsilon\right) \log i+c\right) \mathbb{P}\left\{Y_{n_{c}}=i\right\}+1-2 \mathbb{P}\left\{Y_{n_{c}}=1\right\}\right)= \\
\left.=c+\left(k_{\alpha}+\epsilon\right)\left(\mathbb{E} \log Y_{n_{c}}+\frac{\mathbb{P}\left\{Y_{n_{c}}=n_{c}\right\}}{1-\mathbb{P}\left\{Y_{n_{c}}=n_{c}\right\}}\left(\mathbb{E} \log Y_{n_{c}}-\log n_{c}\right)\right)\right)+ \\
+\frac{1}{1-\mathbb{P}\left\{Y_{n_{c}}=n_{c}\right\}}\left(1-2 \mathbb{P}\left\{Y_{n_{c}}=1\right\}\right)=
\end{gathered}
$$

( $n_{c} \rightarrow \infty$ as $c \rightarrow \infty$; therefore, using (7), the equality $\mathbb{P}\left\{Y_{n}=1\right\}=u_{n-1}$ and the fact that by the elementary renewal theorem $\lim _{n \rightarrow \infty} u_{n}=0$, we obtain)

$$
=c+\left(k_{\alpha}+\epsilon\right) \log n_{c}-\left(k_{\alpha}+\epsilon\right) k_{\alpha}^{-1}+1+o(1) .
$$

Letting $c$ go to $\infty$ leads to $\epsilon \leq 0$, which is a contradiction. Thus, we have already checked that

$$
\varlimsup_{n \rightarrow \infty} \frac{a_{n}}{\log n} \leq k_{\alpha} .
$$

A symmetric argument proves the converse inequality for the lower bound. Therefore,

$$
a_{n} \sim k_{\alpha} \log n
$$

The asymptotic relation 14 for $b_{n}$ can be checked exactly in the same way by using the recursion 13 . Thus, we already know that $\mathbb{E} V_{n}^{2} \sim\left(\mathbb{E} V_{n}\right)^{2}$. Therefore, by Chebyshev's inequality, for any $\delta>0$

$$
\mathbb{P}\left\{\left|V_{n} / \mathbb{E} V_{n}-1\right|>\delta\right\} \leq \frac{\mathbb{D} V_{n}}{\left(\delta \mathbb{E} V_{n}\right)^{2}} \rightarrow 0,
$$

which proves 2 .

In the case when 11 holds with $\alpha=1$, the proof of the fact that $\mathbb{E} V_{n}^{2} \sim\left(\mathbb{E} V_{n}\right)^{2} \sim(\log m(n))^{2}$ is absolutely analogous to the previous one. Thus it is omitted. Certainly, instead of the first part of Lemma 3.1 one has to use the second one.

\section{References}

[1] Bingham N. H., Goldie C. M. And Teugels, J. L. (1989). Regular variation. Cambridge: Cambridge University Press.

[2] ERickson, K. B. (1970). Strong renewal theorems with infinite mean. Trans. Amer. Math. Soc. 151, 263-291.

[3] Feller, W. (1966). An introduction to probability theory and its applications. Vol. 2. New York: John Wiley \& Sons, Inc.

[4] Hinderer, K. AND WalK, H. (1972). Anwendungen von Erneuerungstheoremen und Taubersätzen für eine Verallgemeinerung der Erneuerungsprozesse. Math. Z. 126, 95-115.

[5] IKsanov, A. AND MöHLe, M. (2008). On the number of jumps of random walks with a barrier. Adv.Appl. Prob. 40, 206-228. 
[6] Meyer, P. A. (1966). Probability and potentials. Mass.-Toronto-London: Blaisdell Publishing Company.

[7] Negadajlov, P. (2008). Asymptotic results concernning the absorption times of random walks with a barrier. Prob. Theory and Math. Statist., in press (in Ukrainian). 